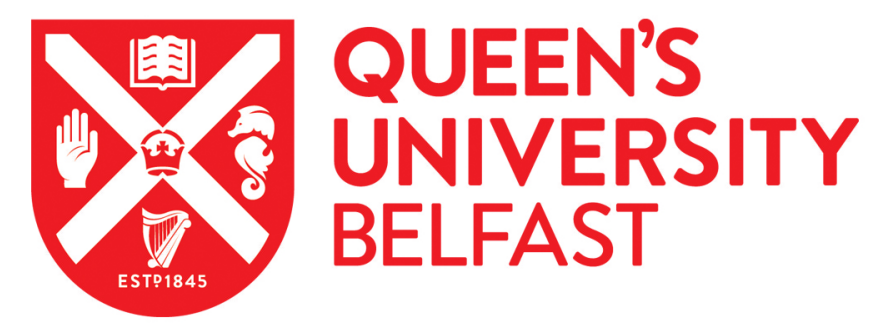

\title{
Distributing Authorship at a Localised Scale: Developing public engagement in sound mapping
}

Mccafferty, C. (2021). Distributing Authorship at a Localised Scale: Developing public engagement in sound mapping. Organised Sound, 26(2), 255-264. https://doi.org/10.1017/S1355771821000297

\author{
Published in: \\ Organised Sound
}

Document Version:

Publisher's PDF, also known as Version of record

Queen's University Belfast - Research Portal:

Link to publication record in Queen's University Belfast Research Portal

\section{Publisher rights}

Copyright 2021 the authors.

This is an open access article published under a Creative Commons Attribution License (https://creativecommons.org/licenses/by/4.0/), which permits unrestricted use, distribution and reproduction in any medium, provided the author and source are cited.

\section{General rights}

Copyright for the publications made accessible via the Queen's University Belfast Research Portal is retained by the author(s) and / or other copyright owners and it is a condition of accessing these publications that users recognise and abide by the legal requirements associated with these rights.

Take down policy

The Research Portal is Queen's institutional repository that provides access to Queen's research output. Every effort has been made to ensure that content in the Research Portal does not infringe any person's rights, or applicable UK laws. If you discover content in the Research Portal that you believe breaches copyright or violates any law, please contact openaccess@qub.ac.uk. 


\title{
Distributing Authorship at a Localised Scale: Developing public engagement in sound mapping
}

\author{
CONOR MCCAFFERTY \\ Queen's University Belfast, Northern Ireland
}

Email: c.mccafferty@qub.ac.uk

\begin{abstract}
Sound maps, particularly the web-based examples that have proliferated since the early 2000 s, have proven compelling and valuable as means of conveying diverse perspectives of urban, rural and wilderness sound environments, while opening the creative process of mapping through field recording to non-expert user groups. As such, sound maps hold the promise of broad public engagement with everyday sonic experience and spatial typologies. Yet this straightforward participatory aim is prone to complication in terms of participatory frameworks and scale of analysis. Drawing on a catalogue of sound maps by the author, this article problematises the participatory norms of sound mapping and, in tandem, calls for a more nuanced approach to scale than typically seen to date in sound maps based on geospatial mapping APIs. A sound mapping workshop in Lisbon with a multidisciplinary participant group provided the opportunity to 're-prototype' sound maps at the scale of a local neighbourhood using multimodal means of representation; the results highlighted questions of form, scale, representation, authorship and purpose in sound mapping and demonstrated its continuing potential as a participatory practice.
\end{abstract}

\section{INTRODUCTION: SOUND MAPS AND SOCIAL ENGAGEMENT IN EVERYDAY SOUND}

Sound maps hold the promise of broad social engagement with everyday sonic experience across a wide variety of spatial typologies. The audio materials that form the texture of these maps certainly include the kind of wilderness and wildlife recordings that have been central to the development of field recording and acoustic ecology. However, more significantly, sound maps encourage recordings of urban, exurban and rural environments by non-expert recordists, with no precedence given to any particular type of environment or recording subject. This creates space for a wide variety of contributions - from smartphone recordings onboard public transport to highfidelity recordings of architectural atmospheres - and leads to projects with multivalent uses and outcomes. As a result of widespread experimentation with sound mapping in the early 2000s by independent artists, collectives and cultural institutions, web-based sound maps proliferated, documenting environments, events and practices of many kinds. In a Catalogue of Sound Maps I produced as part of my doctoral research, I showed how sound mapping as a practice is distributed globally, albeit unevenly so, among hundreds of distinct projects and thousands of contributing participants (McCafferty 2019a). Some sound maps focus on specific cities, towns, neighbourhoods, villages, countries and regions, while others span the entire globe. Cultures and subcultures - from musical to gastronomic - have their own dedicated sound maps. The Catalogue includes sound maps of citizen journalism, folk song, decades-old archival collections, participatory history, birdsong, deep-sea life and wilderness areas. Several of the web-based sound maps that emerged between 2000 and 2020 were shortlived, intentionally or accidentally; it was not uncommon while preparing the Catalogue to find a sound map had disappeared or lapsed into dysfunctionality, presumably for good. Nonetheless, many sound maps have developed and retained engaged participant communities and prioritised their sustainability whether through ongoing institutional support or the personal efforts of (often amateur-enthusiast) practitioners. The concept is still compelling: new sound maps continue to emerge occasionally.

In the past decade, scholars in sound studies, music, art, geography, media studies and other fields have pointed to the relative novelty of web-based sound mapping as new media artefacts, in addition to their potential to form communities of practice and/or interest (see, e.g., Ceraso 2010; Waldock 2011; Radicchi 2012; Bingham-Hall 2014; Carlyle 2014; Ouzounian 2014; Lin 2015; Anderson 2016; Holanda, Rebelo and Paz 2016; Droumeva 2017; Signorelli 2017). Several of these scholars stress the need to engage more critically with sound maps as platforms. The sound art and music scholar Gascia Ouzounian finds in the work of acoustic cartographers an exploratory orientation to the city that actively questions the boundaries of public space (Ouzounian 2014). This interest in the figurative and actual limits 
of sound mapping practice is shared by the sound artist Isobel Anderson, who cautions that 'we must be wary of homogenizing listening in the effort to democratize cartography' (Anderson 2016: n.p.). The media and sound studies scholar Milena Droumeva, meanwhile, positions sound maps in relation to histories of critical cartography but finds that they lack a 'coordinated political aim' (Droumeva 2017: 338).

One feature common to many of the heterogeneous sound maps of recent years is an open call for participating publics. Indeed, these projects often describe themselves using inviting, participatory terms (Waldock 2011: n.p.): social engagement in sound can be seen as simultaneously a key function and a driving goal of sound mapping. Sound maps source their recordings, in principle, from anyone, anywhere, with the means and interest to contribute; as time passes, sound maps accrete layers of audio from this distributed base of contributors. The open source web platforms upon which sound maps are built can be adapted and re-used, and the recordings themselves can be shared directly, or remixed and republished thanks to the flexible licensing they apply to their contents. Engaging with these largescale 'massively open' sound maps as artefacts, the now-ubiquitous visual interfaces of web maps become repurposed as frames to explore the granular detail of auditory experience: 'What was once invisible', sound studies scholar Angus Carlyle notes, 'can be rendered audible' (Carlyle 2014: 141). The flexibility and openness of these interactive projects have many benefits, not least the creation of rich archives of locationspecific audio being made freely available to explore, analyse and repurpose - which highlights the importance of sustainability for sound map projects. In addition to this primary archival purpose, sound maps can be understood - for artists, institutions and audiences - as showcases, co-creative works, alternative historical records, activist platforms, or sensory data repositories. In certain ways, sound maps can also be understood as educational resources, such as for 'teaching listening' - something which, the music scholar Adam Tinkle notes, is central to practices of soundscape studies and acoustic ecology (Tinkle 2015) - and as means of engagement with built environments and spatial typologies through sound (McCafferty 2019b). These latter two points, I believe, have much potential for development in future sound mapping research and practice.

Sound maps have capacity for wide public engagement, cutting across cultural, technical and ecological aspects of sound. But the participatory and spatial frameworks that underpin such projects deserve further scrutiny. It is clear that sound maps offer experienced recordists opportunities to share their work and network with peers; this community of interest is well served by the wide variety of projects now available. For novice participants, however, meaningful participation in the open, web-based form of sound mapping is less obvious: how and why should they contribute? Who is being invited to participate, in the first place? In what follows I consider several challenges of social engagement through sound mapping. If we are interested in meaningful participation among communities of place, we ought to consider more direct and pedagogical approaches to participation that allow active reflection on personal auditory experience alongside the development of skills in listening, recording and mapping. In tandem, as I will discuss later in this article, spatial boundaries that are more clearly defined and localised could produce sound maps of more relevance to communities of place, so that participants can explore common spatial interests through sound.

\section{PARTICIPATORY CHALLENGES, PRECEDENTS AND POTENTIALS IN SOUND MAPPING}

Significant variety can be found in how scholars in different fields conceptualise participation in sound maps. Geographer Wen Lin, for example, describes participation in sound mapping as contributions to the broad set of geographic data, termed 'Volunteered Geographic Information' (VGI) (Lin 2015). This crowdsourced model of participation, usually found in social sciences and geography, depends upon a strong sense of the validity of cartographic expertise: amateur participants are invited to contribute to a well-defined geographic analytical problem with expert support and editorial decision-making. This model does apply in sound mapping in the sense that anyone can 'volunteer' contributions. However, sound maps tend to present users with only a broad spatial limit such as 'the city' or a region. Within that open spatial range, participants can focus on any area, phenomenon, detail or subject that takes their interest. The scale of analysis of the mapping exercise tends to be deliberately broad, and participants' responses are left open to interpretation: sound maps usually offer little guidance about how or what participants might contribute. This open-ended participatory approach parallels the art theorist Claire Bishop's description of relations in participatory (visual) artistic practice: '[T]he artist is conceived less as an individual producer of discrete objects than as a collaborator and producer of situations; the work of art as a finite, portable, commodifiable product is reconceived as an ongoing or long-term project with an unclear beginning and end; while the audience, previously conceived as a "viewer" or "beholder", is now repositioned as a co-producer or participant' (Bishop 2012: 2). Any member of the public, amateur or 
professional, may be a contributor to a sound map, or simply a viewer/listener - or both. As the project continues over time, one's role may alternate between that of audience member and participant.

This unboundedness may be seen as a strength of sound mapping: users can engage with the aspects of the map, spatially or sonically, that most interest them. However, with little prescription over form, duration, quality, recording method, or eventual purpose, questions persist over what participants as coproducers are working to produce. As Droumeva notes: 'Vernacular soundmapping tends to lack a sense of cohesive politics and, while reflective of a plurality of contributions, is not necessarily radical or countercultural' (Droumeva 2017: 338). Droumeva's criticism points to a useful orienting question: what would a cohesive politics around sound mapping look like? The crowdsourced/co-created distinction allows us to reconsider the nature of the participatory engagement: are participants contributing to a predetermined enquiry? Or are they involved in defining and developing the form, content and even the function of the map through their participation?

Questions over cartographic co-production are not limited to sound mapping; they cut across participation in new spatial media more generally. The geographers Sarah Elwood and Agnieszka Leszczynski, for instance, highlight alternative forms of mapping that have multiplied alongside professional GIS-based cartography, fuelled by new spatial media that are accessible to and easily used by 'non-experts in a wide range of everyday practices' (Elwood and Leszczynski 2013: 556). This has led, they argue, to a general de-emphasis of traditional cartographic veracity and verifiability, and a corresponding shift towards witnessing, data sharing, citizen engagement and creative and interactive modes of authorship in contemporary participatory mapping projects (ibid.: 555). In this transformed context, maps have become primarily a conduit for communities of interest to share content that is relevant to their particular cause, issue or political context, to engage in their particular 'knowledge politics' using spatial frameworks. Viewed from this standpoint, the role of sound maps as socially engaging platforms becomes clearer: we can see how sound map creators since around 2000 have provided access to emerging web mapping and audio streaming technologies ('producing situations', to follow Bishop's term, in which publics can participate) and allowed contributors to shape the continually evolving form of the map according to their individual or collective interests. Participants can share moments of sonic interest and surprise, or showcase their technical skill, usually with few restrictions on form or content. The results can be read as musically (as much as geographically) experimental. Still, the technical sophistication of linking streaming audio to specific locations ought to encourage reflection: what might these collections of geolocated sound reveal about urban spaces that other maps do not, or cannot? It is a question that sound maps tend to ignore or avoid. Despite a flurry of experimentation with technologically innovative and satisfying sound maps, something may have been lost. Some earlier experiments with sound mapping provide useful insights into both participatory practice and the visual and graphic potentials of cartography.

Sonic World (Sonarchy Collective, 1997-2006) ${ }^{1}$ can be seen as a precursor to present-day web-based sound maps that use a participatory model of content creation. The project employed a conceptual approach that was similar to later sound maps, but it did not take the same form, and its collection never expanded beyond a few dozen recordings. Visitors to the site could browse a collection of sound files that had been recorded at sites around the world, presented alongside images and commentary. The Sonic World homepage consisted of the project logo with a prominent button labelled 'Destinations': clicking this, users could explore the site's content. Instead of using one central base map as the means of orientation and interaction with content, entries were organised geographically in lists by continent, country and site (e.g., Africa - Egypt - Valley of the Kings). Each entry was hosted on an individual page of the website, with text commentary, a map image and links to download recordings in RealAudio and Wav format.

The production of a sound map even 20 years ago constituted a markedly different task than it does today. The development of Sonic World in the late 1990s meant that it narrowly missed the emergence of widely available streaming media and web mapping, both of which have been key to the subsequent evolution of online sound maps. Like later sound maps, Sonic World invited participants to submit content, but since the producers of the website and its visitors were restricted in terms of storage space and bandwidth, it sought mono or stereo sound files of no more than 30 seconds in length, which contributors could submit by FTP or by post on cassette, DAT or reel-to-reel tape. Today, with the combination of nearubiquitous high-speed internet access and capacious cloud-based data storage, these heritage media formats might strike us as quaint. Content creators can now upload multimedia files many factors larger with

${ }^{1}$ Sonic World is no longer active but its content, including several of its audio files, can still be accessed via the Internet Archive's Wayback Machine at http://web.archive.org/web/20060207090133/ http://www.sonarchy.org/sonicworld/ (accessed 15 September 2020). This archival website contains backups for Sonic World dating from 1997 to 2006, when we can presume the site went offline. 
little delay, often using the same handheld smartphone device to both create and upload digital artefacts. From the front-end of websites and in smartphone apps, users can download or stream large packets of multimedia content instantaneously, without leaving the web browser or having to navigate folder structures, as part of a 'frictionless' user experience of the Web.

Yet while its visual style and technological base have been quickly outmoded by advancements in web and recording technology, Sonic World remains instructive for a few reasons. At a time when users had to consciously download content to their hard drive and open it in a media player application to listen, the producers of the sound map limited the maximum length of participants' contributions, arguing that 30 seconds was ample time to convey a sense of place. The notion that 30 seconds of audio can 'concisely convey the essence of a location' (Sonarchy Collective, 'Your Ambience is Requested' 1999: n.p.) may be contested. ${ }^{2}$ However, the producers of Sonic World used the technical limitation on recording length to pose a creative challenge to their participants.

In Sonic World, the onus was on contributors to present a personal experience of listening and to convey to others a sense of place through the materials they contributed to the project. Contributors would use a sound recording, a text narrative and their choice of 'map' image to represent their chosen location effectively collaging multimedia content. Each 'destination' had its own page on the website, presenting the contributor's text narrative and chosen image alongside the recording. By giving contributors responsibility for the choice of map, the producers of Sonic World saw a way to deepen the creative experience of the project: 'We want to leave this request open-ended because it is an opportunity for some creativity on your part. It could be hand drawn or lifted from an atlas. The perspective is up to you' (ibid.: n.p.). The process of 'sound mapping' in Sonic World consisted of a multimodal creative act and thus adopted a markedly different approach to that seen in more recent webbased sound maps, where every contributor engages with the same base map interface to geolocate recordings. Drawing from traditions of multimedia art, Sonic World not only promoted creative audio recording and production but also invited participants and audiences to reflect on personal auditory experience through cartographic visuals, text and audio, producing a rich collage of diverse sounds, written reflections and map images from multiple perspectives.

${ }^{2} \mathrm{~A}$ later sound map, the LocusStream Sound Map (Locus Sonus 2006-) provides an interesting counterpoint: it features live 'open mikes' broadcast continuously from locations around the world, allowing users to listen as long as they wish, or to revisit the same location multiple times. Such sustainable long-termism is notably absent from many sound map projects.
The focus on subjective and multivalent responses to sound in Sonic World serves as an important reminder that apparently neutral acoustic phenomena will always be coloured by a person's experiences, memories and learning, by the physical capacities of her perceiving body and by the culture and society that she inhabits. Jonathan Sterne calls for a rejection of a 'creeping normalism' (Sterne 2015: 73) that he perceives in sound studies: 'We should hold onto the idea that the ways people can hear, the limits of that hearing, and the conditions of possibility for hearing all provide points of entry into what it means to be a person at a given time or place' (ibid.: 73). Although they also comprise contributions from multiple users, contemporary sound maps, by contrast, do not emphasise the individuality of participants' contributions. Instead, these later examples typically foreground the stream of participants' recordings as the primary feature of the sound map. User interfaces in contemporary sound maps continually hold out the possibility to switch to another audio stream, or even, in some cases, to mix audio from different locations. This emphasis on listening and the skilled practice of field recording in sound maps creates valuable archives of recordings, presented in an engaging way; however, we should consider what aspects of personal experience may be downplayed or overlooked in this approach, and how the involvement of people of different backgrounds might transform collective sound mapping exercises.

Earlier still, a compelling example of distributed agency in mapping urban sound can be found in a 1969 study of the 'sonic environment' of Boston by the urban planner Michael Southworth. His article 'The Sonic Environment of Cities' featured maps and illustrations of the city that can be seen as an important precedent for later sound mapping, even if his singular approach, rooted in social science, has not been replicated since. Southworth's study used a distributed approach to mapping where content derives from the auditory experience of residents (who were blindfolded and pushed around predetermined routes in the city in wheelchairs) expressed through a symbolic visual syntax. Southworth suggested new ways of understanding sound as a component of urban space that could, crucially, be analysed and characterised in tandem with visual phenomena (Southworth 1969).

More than 50 years have passed since Southworth conducted his study of Boston's sonic environment, yet his foregrounding of subjective auditory experience in an urban studies context remains something of an anomaly. In response to legislation and an increasing focus on public health, city authorities have been successful in attenuating harmful levels of noise using the noise abatement approach, and the accumulation of statistics on urban noise levels - often now using $\mathrm{AI}$ and machine-learning, as urban media 
scholar Shannon Mattern points out (Mattern 2020: n.p.) - offers a useful baseline asset for urban sound research. Yet cities around the world continue to encounter the kinds of problems - relating not only to excessive noise but equally to a lack of 'sonic identity' - that Southworth identified. For Southworth, a sonic identity, based on the 'uniqueness' and 'informativeness' of a given sound setting (Southworth 1969: 54), connects acoustic experience to a sense of place. Southworth's reassessment of urban space in terms of sound has been bolstered in subsequent years by empirical research from diverse quarters, such as the richly detailed catalogue of urban sonic effects by Jean-Franćois Augoyard and Henri Torgue (Augoyard and Torgue 2005) and sound artist Peter Cusack's concept of 'sonic places' (Cusack 2017). Auditory experience is an essential element of any designed environment, as the scholars of psychology and space Barry Blesser and Linda-Ruth Salter note: 'In each contrasting space, even if the sound sources were to remain unchanged, the aural architecture would change. Every space has an aural architecture' (Blesser and Salter 2006: 2). Decisions by designers - and the exigencies of urban development fundamentally impact the acoustic qualities of the built environment. In addition to their valuable role as hubs for field recording, I suggest that sound maps can have other practical roles. Sound maps - if considered in the context of specific, directed mapping inquiries at localised scales - have the potential to bring heightened auditory awareness to those who use and design buildings and urban spaces, and thus to make sound a more tangible object of consideration in built environment practice. In this context, the potential emerges for collaborative, interdisciplinary sound mapping: field recordists, sound artists, composers and acoustic ecologists can take a guiding, pedagogical role among a broader field of practitioners including architects, urban designers, community artists and activists. Yet we cannot ignore barriers in implementing such an interdisciplinary approach to sound mapping, whether encouraging researchers and professionals to collaborate across disciplinary boundaries ${ }^{3}$ or engaging novices with sonic practice. Removing such barriers may lie within the scope of existing sound map projects or in developing new forms of the practice. In what follows, I will first consider how sound maps can offer instructional guidance to participants, before discussing a workshop that invited participants of various disciplinary backgrounds to develop new prototype sound maps at the local scale of a small urban neighbourhood in Lisbon.

${ }^{3}$ For a discussion of one disciplinary impasse, see Michael Fowler's article on the bifurcation of acoustics and architectural design (Fowler 2015).

\section{SHARED MAPS - SHARED LISTENING}

For a practice that seeks to engage participants with sound, primarily through field recording, ${ }^{4}$ there is little acknowledgement in most web-based sound map projects of the complexity of field recording, its technical and creative potential - and its pitfalls and challenges. Most of the instructional guidance to be found in sound maps is relatively basic or relies on outside sources. One way to maintain a particular standard of field recording in sound maps - especially relevant when material is contributed remotely - is to provide guidelines to prospective participants, such as those presented on the 'About' page of the radio aporee sound map project founded by Udo Noll (2008-). The project lists explicit 'rules' for the kind of content that it seeks. When prospective participants click the 'add a new location' button, a reminder appears asking them to 'respect the posting rules' before uploading, with a link to the list of rules. The rules request participants to commit seriously to their participation in the project and to contribute highfidelity recordings that are focused on a single specific location. The rules are intended as a method of gatekeeping, protecting radio aporee as a space for 'quality' field recordings. Noll offers a terse summary in bold, red letters at the beginning of the list: 'tl; $\mathrm{dr}$ make \& post field recordings of decent quality!' (ibid., italics in original). The rules are didactic in tone: 'The sounds we want to hear are field recordings, sounds from actual sites and situations, including their surrounding ambiences' (ibid.:, n.p.); Noll warns users against contributing 'mobile phone recordings', 'songs', 'soundart creations' or 'spam' (ibid.: n.p.). Yet rather than elaborate on the nuances of site-based, ambient field recording, Noll moves on to list other websites and resources that introduce field recording practice. Nonetheless, these broad rules of engagement provide a useful starting point for participants - and are more detailed than most sound maps tend to offer. They also touch upon a significant ethical issue that any recordist must face - namely, the recording of voices or conversation and gaining consent from subjects when necessary. We can surmise that this location-focused approach to field recording generally considers human voices to form part of the 'surrounding ambience' of a site - one acoustic feature among many. Still, it is imperative that recordists can judge the recording context and draw clear distinctions between vocal ambiences without discernible speech and recordings that directly convey private conversations, produced intentionally or inadvertently. Noll

${ }^{4}$ There are some notable exceptions to the use of field recording in web-based sound maps, such as the facility on the Belfast Sound Map (Rebelo, Chaves, Meireles and McEvoy 2012-) to contribute a 'text description' of a sound experience. 
advises contributors: 'take care about privacy, e.g. don't record private conversations etc. without permission' (ibid.: n.p.).

While some sound maps, including radio aporee, have assumed the role of maintaining standards of field recording and introducing new generations to field recording practice, sound mapping broadly remains a locus of experimentation. As well as highlighting commonalities, the Catalogue of Sound Maps reveals numerous features that are distinctive in specific sound maps. As new iterations of sound maps have appeared, new approaches and new concepts build on what came before. The functions, features and aims of sound maps continuously go through re-prototyping to meet the needs and interests of particular communities and the imperatives of research and artistic processes.

I explored this re-prototyping in a two-day workshop I was invited to deliver in Lisbon, Portugal in February 2020 as part of Sounds of Tourism, a research project based at Lisbon's NOVA FCSH university. ${ }^{5}$ Lisbon was a city that I already knew well: having experienced it as both a visitor and a researcher several times, I was familiar as an outsider with its evolution over more than a decade, during which time I witnessed its ever-increasing popularity as a tourist destination. However, aside from browsing the Lisbon Sound Map (Ribeiro, Hélin, Mota, Bruno, Pinto and Jorge 2013-) online, this was my first experience of Lisbon in terms of researching urban sound, and I had not previously been to Mouraria, the neighbourhood in which the workshop took place. (Mouraria was purposely selected by the Sounds of Tourism project because it has been going through major change and gentrification in recent years; a longer programme of community-based research in Mouraria led by Sounds of Tourism researchers preceded the workshop and has continued afterwards.) The workshop drew upon the networks of the Sounds of Tourism project and invited people with different artistic, scholarly or professional expertise to explore urban sound mapping. Although no prior experience or qualification was required, participants self-selected: they expressed an interest in this part of the city, in sound mapping or in sound art more broadly. A group of 30 people took part; while several had backgrounds in sound studies or music, there was a broad disciplinary mix, including journalists, anthropologists, architects, artists and scholars of urban studies (Figure 1). The workshop offered an opportunity to explore certain specific concerns of my sound mapping research with the group: a twodecade history of web-based sound maps; sound

${ }^{5}$ www.soundsoftourism.pt/; information specifically about the workshop can be found at www.soundsoftourism.pt/urban-soundmapping-practices/ (accessed 17 December 2020). mapping at localised, specific urban scales; the sharing of multidisciplinary perspectives of sound; and the use of multimodal means of representing sound experience that could complement and co-exist with field recording. For participants, the workshop was an opportunity to learn about practices of sound mapping, to network with people of different backgrounds who shared an interest in urban sound and to prototype their own sound map of an urban neighbourhood. As I will show, the results were rich and varied, but there are of course limits to what can be achieved in a brief two-day workshop. As a limited and focused exercise with a group of interested participants, the workshop can be seen as a pilot study that sought to suggest new possibilities of sound mapping (both for this location and in general). Given further time and resources, a longer-term approach that engages local residents alongside professionals and researchers in the co-design of a localised sound mapping process, built upon existing sound map platforms or on a bespoke platform, could yield valuable new insights through socially engaged practice. ${ }^{6}$

The workshop constrained the scale of the group's practical work to Mouraria, which displays a broad cultural mix reflective of its demographics: the area is home to a diverse population of long-term Lisbon residents (mostly older people) alongside ethnic minority groups who have more recently moved here on arrival to the city, in addition to transient city dwellers and tourists who are accommodated in new apartments and refurbished older buildings. Close to the city centre of Lisbon, the area is impacted by the tourism industry and has become a popular destination for visitors, but it remains less commercialised and more residential than some other nearby areas. Participants from different parts of the city, from outside Lisbon and, in some cases, from outside Portugal came together in this workshop to explore this dense and diverse urban milieu. Some participants came with previous academic, professional or personal experiences of the area, some had preconceptions without being familiar, while others, including me, knew little about it prior to the workshop. ${ }^{7}$

After presenting a condensed history of sound mapping practices and technologies in a global context, I invited the group to consider sonic experience on a local level. Before venturing outside the Mouraria Creative Hub, the venue that hosted the workshop, the participants began using sonic imagination to

${ }^{6}$ For an example of a longer-term, community-embedded sonic methodology, see Linda O'Keeffe's study of Smithfield, Dublin, which she developed with young people living in the area (O’Keeffe 2015)

${ }^{7}$ I purposely avoided researching the area in too much detail, so that I would not influence the participants with my own conceptions. 




Figure 1. Participants in the Lisbon Urban Sound Mapping workshop discuss the range of sounds they expect to record in the neighbourhood of Mouraria. Photograph by Iñigo Sánchez-Fuarros, courtesy of the Sounds of Tourism project.

inform their approach to the mapping process. In five independent groups, the participants proposed sounds they felt might convey a sense of the area, and which they could include on their prototype sound maps of Mouraria. Without wanting to constrain their responses, I suggested some initial prompts, including 'signature sounds' of the area (such as the sounds of local events and activities), the sounds of locations of interest, interiors and exteriors, and sound in architectural and urban spaces. This session encouraged participants to reflect on both the subject area and the practices of listening and recording they might use in their prototype.

When thinking about possible events and phenomena they might record, some groups had specific locations in mind, including Graça church, street markets, a local shopping centre, the Miradouro da Graça (a local viewpoint that attracts groups of residents and tourists) and the Clube Desportivo de Mouraria (a local sports and social institution). Others wanted to explore general spatial typologies such as courtyards and streets, squares, shops, cafes, staircases and alleyways and details such as the textures of paving and domestic sounds. Some expected to hear construction sounds since the area was in transformation. Several of the groups mentioned listening out for voices, conversations, the sounds of children, and people 'thinking aloud' on the streets, which they felt would highlight the multicultural demographics of Mouraria; similarly, different languages and 'touristic voices' might show the impact of tourism on this part of the city. (To address the ethical concerns around recording speech, all participants in the workshop followed the principle that discernible voices or conversations should only be recorded with the informed consent of the subject. Recordings were only shared in the context of the workshop itself, and not made public later.) Groups whose members had music and sound art backgrounds identified musical concepts and specific recording techniques as a means of exploring the area - such as electro-magnetic sounds, spaces with interesting acoustics, music performances in urban space, church bells, doorbells and the bells of trams, the rhythms of footsteps and sounds at high and low frequencies. Several groups hoped to record the sounds of nature and wildlife: birds ('both caged and free'), cats, dogs and 'others', gardens, parks and trees. Others wanted to capture the various forms of urban transportation that traversed the area: trams, metro, cars, bikes and scooters. Even this preparatory activity began to illuminate various spatial, architectural and social characteristics of Mouraria, while encouraging a sense of shared purpose among participants. Following these discussions, each group prepared a collection plan and set about gathering materials for their maps that evening and the following morning.

On the second day of the workshop, the groups began organising the materials they had collected. 
The brief purposely avoided any technological preference. I offered suggestions of existing mapping platforms that could be used, but also encouraged experimentation with form and functionality. A drawing, a performance or an annotated photograph had as much value as a field recording. Indeed, rather than thinking of a sound map as a collection of 'pinned', geolocated recordings, the groups had to consider what presentational approaches would allow them to build a proof of concept and show their findings relatively quickly. It was striking that under such circumstances, and under time pressure (they had only an afternoon to sort through and prepare their materials for presentation), groups demonstrated a wonderful variety of approaches that ranged from slideshows to fully fledged interactive web maps with streaming audio.

After completing their creative work, each group discussed the concept that lay behind their prototype sound map and played samples of their recordings. One group, which included two anthropologists, talked about their attempt to uncover local perspectives by listening out for people's voices on the street and engaging people in conversations. For example, it is common in Mouraria for residents to lean out of their upper-floor apartment windows, spending time observing the street below; in one recording, with the subjects' permission, the group listened in on a discussion between two local women at the windows of their apartments, facing each other across a street, socialising and discussing local events, their voices gently echoing against the hard surfaces around them. Presented alongside a simple pen and paper sketch of the buildings and the women at the windows, this short recording effectively expressed one important aspect of spatial and social relations in the neighbourhood. The same group also presented a recording of a local resident, made with his consent, who gave his opinions about gentrification and the transformations he was witnessing in the area. This group and others also took time to find spaces of relative quiet in the area, recording calmer soundscapes away from tourist hotspots and heavily traversed streets and junctions.

For other groups, freely available web mapping platforms offered a way to organise and present their recordings using existing spatial frameworks. One group used a standard point-and-click style sound map interface to present a selection of their numerous recordings of interior and exterior spaces in Mouraria. The fact that a viable proof-of-concept can be deployed in the course of an afternoon using existing tools and web services illustrates the advancements in web streaming and geolocation technologies in the years since web-based sound maps first emerged. That is not to detract, however, from the ingenuity and skill this group showed in presenting their material in this way in such a short space of time. Another group used a similar multimedia mapping platform to present each group member's route to the workshop venue from their respective home in Lisbon. The walks, collaged into a few minutes' audio, illustrated diverse experiences of traversing the city and into Mouraria from several directions. The final group used a web map only to show the route they followed to make their recordings and observations; they presented their sounds accompanied by photographs they had taken at each recording location. Their recordings sought out rhythmic and melodic variety, from the less obvious, such as rumbles of traffic over cobbled streets, to the better-known: the ballad 'Saudade' performed at sunset on the Miradouro da Graça.

Buildings and urban spaces of great variety, everyday sociality, musical interpretations of the soundscape: over the course of two days in Mouraria, this workshop amassed a variety of material portraying sonic experiences using multimodal means and demonstrated how practices of listening and recording allow unique perceptions of place to surface. The workshop showed how distinctive disciplinary interests could merge in sound to build a rich, multimodal representation of a single neighbourhood at a specific moment in time. Crucial to the development of these sound maps were exercises in sonic thinking, the development of multidisciplinary approaches to mapping, and debate and collaboration within and between groups. The thought-provoking and engaging results, musical, ecological and anthropological, emphasise the potential for such co-creative interdisciplinary sound mapping in urban sonic research. For this potential to grow, new or hybrid forms of sound mapping platforms may be required, but equally, existing sound maps may have the capacity to house such forms of interdisciplinary work in their local context and to realise more strongly their promise of participatory community engagement.

\section{CONCLUSION: TOWARDS SHARED LISTENING IN SOUND MAPPING}

To fulfil the participatory promise of sound mapping requires a commitment to maintaining the existing web-based forms of the practice and sustaining the rich archives they have amassed, while continuing to explore social engagement with the practice in local contexts. The 'standard' approach to web-based sound mapping distributes the creation of content among many participants, but often lacks social engagement in that process. While the accumulative-archival works that result contain numerous interesting and often remarkable recordings, the benefits of opening reflective processes of sound mapping to broader audiences remains underexplored. 
The experience of the workshop in Lisbon crystallised ideas I had explored in my doctoral research regarding urban sound mapping in contexts of urban development. First, the workshop confirmed the usefulness of a 'localised' approach to scale in urban sound mapping. In existing interactive sound maps, the problem arises that recordings of many different scales sit alongside one another on a single map of the city. Each individual recording articulates an idiosyncratic scale that does not always bear relation to the map's visual appearance. I suggest, instead, that the enquiry to be pursued through sound mapping should dictate the spatial scale for recordings and any other material contributed to the map. The scale of the sound map should be localised and specific: recordings should respond to the scale of the map, rather than the other way around. 'Local' is a relative term: a local scale could comprise an architectural site, a small urban neighbourhood (as in the case of Mouraria) or district, or a predetermined route through a city. A scale is 'specific' in the sense of being specified in advance - and, where possible, negotiated with participants as part of the sound mapping exercise.

Second, the wide variety of materials produced by the workshop participants demonstrated the range of possible techniques for 'listening to the city' ${ }^{8}$ In this context, the use of a wider range of material beyond field recording does not simply serve some arbitrary directive to use multimedia content. Rather, it is foremost a question of legibility of the sound environment across and between disciplines of sound art and architecture, and beyond: the map ought to be readily understood whether one has technical expertise in sound or the built environment, both, or neither. For example, while the timbral quality of an early evening soundscape might be best represented in a field recording, a drawing of the layout of structures and buildings on site could be a more immediate way to account for the echoes of a bird call that contributes to that ambience. Thus, the sound map need not consist of either field recordings or other materials, but bothland.

I propose a socially oriented approach to sound mapping in which communities of place take priority and ownership over the mapping process, such that multidisciplinary communities of interest may work collaboratively within it. Such an approach seeks to open sonic practices to those who may benefit from engaging with them. In this light, the various forms sound mapping I have outlined, and exploratory workshops such as the one I discussed here, can be seen as precursors to longer term socially engaged

${ }^{8}$ See Williams (2017) for a collection of techniques and prompts to help guide practices of 'listening to the city'. sonic practices that are embedded within a community and that can offer benefits to that community, whether as creative outlets, archival repositories or rallying points for community activism. In our works of urban cartography, Shannon Mattern urges us to remember 'the value of mapping as a method and of thinking of the map itself as a medium to which we have to apply our critical faculties' (Mattern 2015: 49). The ingenuity of cartographic design over centuries has shown us the sheer variety of what we might focus on in mapping cities, and the lives led in them. Sound mapping, as a socially engaged sound practice, provides a useful reminder of the worlds of meaning, detail and connection that lie behind the map's static point.

\section{Acknowledgements}

Parts of this article have been developed from my doctoral thesis (McCafferty 2019b). I would like to thank Dr Iñigo Sánchez-Fuarros for inviting me to deliver the workshop in Lisbon, the journal reviewers and Dr Matilde Meireles for their constructive advice and feedback on earlier drafts of the article, and my colleagues in the Recomposing the City research group for their support. Sincere thanks to all the workshop participants who gave so generously of their time and ideas and contributed to a memorable two days in Lisbon.

\section{REFERENCES}

Anderson, I. 2016. Soundmapping Beyond the Grid: Alternative Cartographies of Sound. Journal of Sonic Studies 11. www.researchcatalogue.net/view/234645/ 234646/0/0 (accessed 15 September 2020).

Augoyard, J. F. and Torgue, H. (eds.) 2005. Sonic Experience: A Guide to Everyday Sounds. Montreal: McGill-Queen's University Press.

Bingham-Hall, J. 2014. How Now Sound City? Blog, Public Culture. http://johnbinghamhall.tumblr.com/ post/101514047569/how-now-sound-city (accessed 15 September 2020).

Bishop, C. 2012. Artificial Hells: Participatory Art and the Politics of Spectatorship. London: Verso.

Blesser, B. and L.-R. Salter. 2006. Spaces Speak, Are You Listening? Cambridge, MA: MIT Press.

Carlyle, A. 2014. The God's Eye and the Buffalo's Breath: Seeing and Hearing Web-Based Sound Maps. Proceedings of Invisible Places/Sounding Cities: Sound, Urbanism and Sense of Place, Viseu, Portugal, July, 141-52.

Ceraso, S. 2010. The Sight of Sound: Mapping Audio. Blog, HASTAC: Humanities, Arts, Science and Technology Alliance and Collaboratory. www.hastac.org/blogs/ stephceraso/2010/10/05/sight-sound-mapping-audio (accessed 15 September 2020).

Cusack, P. 2017. Berlin Sonic Places: A Brief Guide. Berlin: Wolke Verlagsges. 
Droumeva, M. 2017. Soundmapping as Critical Cartography: Engaging Publics in Listening to the Environment. Communication and the Public 2(4): 335-51.

Elwood, S. and Leszczynski, A. 2013. New Spatial Media, New Knowledge Politics. Transactions of the Institute of British Geographers 38: 544-59.

Fowler, M. 2015. Sounds in Space or Space in Sounds? Architecture as an Auditory Construct. Architectural Research Quarterly 19: 61-72.

Holanda, C., Rebelo: and Paz, A. 2016. Soundmaps as iDocs?: Modes of Interactivity for Storytelling with Sound. Leonardo Music Journal 26: 80-2.

Lin, W. 2015. The Hearing, the Mapping, and the Web: Investigating Emerging Online Sound Mapping Practices. Landscape and Urban Planning 142: 187-97.

Locus Sonus Collective. (2006-) Locustream SoundMap. http:// locusonus.org/soundmap/040/ (accessed 15 September 2020).

Mattern, S. 2015. Deep Mapping the Media City. Minneapolis: University of Minnesota Press.

Mattern, S. 2020. Urban Auscultation; Or, Perceiving the Action of the Heart. Places Journal, April. https:// placesjournal.org/article/urban-auscultation-or-perceivingthe-action-of-the-heart/ (accessed 18 August 2021).

McCafferty, C. 2019a. Catalogue of Sound Maps, 19972018. Recomposing the City. www.recomposingthecity. org/soundmaps (accessed 15 September 2020).

McCafferty, C. 2019b. Urban Sound Mapping in Sound Art and Built Environment Practice. PhD thesis, Queen's University Belfast.

Noll, U. (2008-) About Content. Radio Aporee. https://aporee. org/maps/info/\#content (accessed 18 August 2021).

O'Keeffe, L. 2015. Thinking Through New Methodologies. Sounding Out the City With Teenagers. Qualitative Sociology Review 11(1): 6-32.
Ouzounian, G. 2014. Acoustic Mapping: Notes from the Interface. In M. Gandy and B. Nilsen (eds.), The Acoustic City. Berlin: Jovis. 164-73.

Radicchi, A. 2012. Sull'immagine sonora della citta. Florence: Firenze University Press.

Rebelo, P., Chaves, R., Meireles, M. and McEvoy, A. 2012. Belfast Sound Map. http://belfastsoundmap.org (accessed 15 September 2020).

Ribeiro, L. C., Hélin, M., Mota, H., Bruno, J., Pinto, J. G. and Jorge, R. P. (2013-). Lisbon Sound Map. www. lisbonsoundmap.org (accessed 15 September 2020).

Signorelli, V. 2017. Listen Through the Map: Role and Improvements of Digital Cartography in Exploring the Urban Sonic Environment. In B. Piga and R. Salerno (eds.), Urban Design and Representation: A Multidisciplinary and Multisensory Approach. New York: Springer International. 153-64.

Sonarchy Collective (1997-2005). Sonic World. http://web. archive.org/web/19970506083631/www.sonarchy.org/ sonicworld (accessed 15 September 2020).

Southworth, M. 1969. The Sonic Environment of Cities. Journal of Environment and Behaviour 1(1): 49-70.

Sterne, J. 2015. Hearing. In D. Novak and M. Sakakeeny (eds.), Keywords in Sound. Durham: Duke University Press, 65-77.

Tinkle, A. 2015. Sound Pedagogy: Teaching Listening since Cage. Organised Sound 20(2): 222-30.

Waldock, J. 2011. Soundmapping: Critiques and Reflections on this New Publicly Engaging Medium. Journal of Sonic Studies 1. www.researchcatalogue.net/view/ 214583/214584 (accessed 15 September 2020).

Williams, A. (ed.) (2017). Listening to the City: Community Research and Action Through Sound and Story. www. colab.mit.edu/resources-1/2018/4/2/listening-to-the-cityhandbook (accessed 15 September 2020). 